\title{
Mass formulae and staticity condition for dark matter charged black holes
}

\author{
Marek Rogatko ${ }^{\mathrm{a}}$ \\ Institute of Physics, Maria Curie-Sklodowska University, pl. Marii Curie-Sklodowskiej 1, 20-031 Lublin, Poland
}

Received: 30 July 2020 / Accepted: 2 September 2020 / Published online: 21 September 2020

(C) The Author(s) 2020

\begin{abstract}
The Arnowitt-Deser-Misner formalism is used to derive variations of mass, angular momentum and canonical energy for Einstein-Maxwell dark matter gravity in which the auxiliary gauge field coupled via kinetic mixing term to the ordinary Maxwell one, which mimics properties of hidden sector. Inspection of the initial data for the manifold with an interior boundary, having topology of $S^{2}$, enables us to find the generalised first law of black hole thermodynamics in the aforementioned theory. It has been revealed that the stationary black hole solution being subject to the condition of encompassing a bifurcate Killing horizon with a bifurcation sphere, which is non-rotating, must be static and has vanishing magnetic Maxwell and dark matter sector fields, on static slices of the spacetime under consideration.
\end{abstract}

\section{Introduction}

One of the long-standing puzzle of the contemporary physics and astronomy is the dark matter problem, ingredient which constitutes over $23 \%$ of the observable Universe, being an important factor for the construction of cosmic web on which the ordinary matter accumulate $[1,2]$.

Nowadays dark matter sector interaction with the Standard model particles $[3,4]$ is widely elaborated. Several new types of fundamental particles were claimed to be the candidates for the hidden sector. They are expected to interact with nuclei in detecting materials on Earth [5-7]. On the other hand, there is a resurgence of implementing physics beyond the Standard Model to explain dark matter interaction in non-gravitational sector [8-10].

The studies of gamma rays emission in dwarf galaxies, oscillation of the fine structure constant, exploration of the possible low-energy mass dark sector [11-13], studies of dark photon production in the former observations of super-

a e-mails: marek.rogatko@ poczta.umcs.lublin.pl; rogat@kft.umcs. lublin.pl (corresponding author) nova 1987A event [14], Gaia-like astrometry observations [15], as well as, studies of dynamics of galaxy clusters collisions [16], are expected to deliver new way of elaborating the old problem. Recently SENSEI device [17] is dedicated for the direct-detection of dark matter in $\mathrm{eV}$ to $\mathrm{GeV}$ energy range. It is expected that it provides constraints on dark matter electron scattering and on dark photon absorption of electrons.

However, the absence of evidences of the most popular particle candidates for the dark matter sector is affirmed, in some sense, as a dilemma in the hidden sector researches. It has been proposed [18] that one should carefully analyse upcoming astrophysical phenomena, such like black hole and neutron star properties, gravitational wave signals to convey complimentary information connected with the dark matter sector.

The problem in question, justifies also our researches connected with the influence of dark matter sector on black hole physics. In what follows we shall try to find the mass formulae for black holes (especially effect of hidden sector on it), as well as, to prove the staticity theorem for black holes in dark matter gravity. As the staticity theorem imposes the additional conditions on the dark matter field, we suppose that the results will be of interests in further inspection of dark sector properties.

In our work we shall consider the model of dark matter sector in which the additional $U(1)$-gauge field is coupled to the ordinary Maxwell one. This the so-called dark photon model. The action describing Einstein-Maxwell dark matter gravity $[19,20]$ is provided by

$S=\int d^{4} x \sqrt{-g}\left(R-F_{\mu \nu} F^{\mu \nu}-B_{\mu \nu} B^{\mu \nu}-\alpha F_{\mu \nu} B^{\mu \nu}\right)$,

where $R$ is the spacetime scalar curvature, $g_{\mu \nu}$ is fourdimensional metric tensor, $\sqrt{-g}$ stands for the square root 
of its determinant, while $F_{\mu \nu}=2 \nabla_{[\mu} \tilde{A}_{\nu]}$ is the ordinary Maxwell field, $B_{\mu \nu}=2 \nabla_{[\mu} \tilde{B}_{\nu]}$ is the additional $U(1)$ gauge field, mimicking the dark matter sector coupled to Maxwell one. The auxiliary $U(1)$-gauge field is coupled to the Maxwell one by means of the so-called kinetic mixing term with a constant $\alpha$. The predicted value of $\alpha$, stemming from realistic string theory compactifications is between $10^{-2}$ to $10^{-16}$ [21-24]. The model described by the action (1) has its roots in string/M-theory, where the mixing portal arises quite naturally in open string theory. On the other hand, in supersymmetric Type I, Type II A, Type II B models, both gauge states are supported by D-branes separated in extra dimensions [25].

In the action (1) the auxiliary gauge field is connected with some hidden sector [25]. In [26] such model was used to describe the existence and subsequent integrating out of heavy bi-fundamental fields charged under the $U(1)$-gauge groups. In general, such kind of terms emerge in the theories having in addition to some visible gauge group, the other one in the hidden sector. Such scenario is realised e.g., in compactified string or M-theory solutions generically possess lit hidden sectors which contain the gauge fields and gauginos, due to the various group factors included in the gauge group symmetry of the hidden sector.

The hidden sector in the low-energy effective theory contains states which are uncharged under the the Standard Model gauge symmetry groups. They are charged under their own groups and interact with the visible ones via gravitational interaction. We can also think out other portals to our visible sector [27,28]. For the consistency and supersymmetry breaking [29], the realistic embeddings of the Standard Model in $E 8 \times E 8$ string theory, as well as, in type I, IIA, or IIB open string theory with branes, require the existence of the hidden sectors.

As far as the possible experimental justification of the aforementioned theory is concerned, it happens that astrophysical observations of $511 \mathrm{eV}$ gamma rays [30], experiments detecting the electron positron excess in galaxies [31-33], as well as, possible explanation of muon anomalous magnetic moment [34], strongly advocate the presented idea. The kinetic mixing term between ordinary boson and relatively light one (the dark one) described as $U(1)$-gauge symmetry and connected with a hidden sector, may cause a low energy parity violation [35], which envisages itself in the Higgs boson decays, and production of a relatively light vector boson with mass $m \geq 10 \mathrm{GeV}$ [36].

On the other hand, black holes are the other objects that still acquire attention in general relativity and its generalisations. The main motivation for our work is to elaborate how dark matter sector influences black hole physics and characteristics like mass, angular momentum. We shall also consider how the the hidden sector modifies the so-called staticity theorem which on its side constitutes the key start- ing point for the uniqueness theorem for black holes in the theory under consideration.

The staticity theorem was attributed to Lichnerowicz, who proved that a stationary fluid was everywhere locally static, i.e., its flow vector aligned with the Killing timelike one, was static being the hypersurface orthogonal [37]. Next extension was given in [38], where the vacuum non-rotating black holes were considered in the sense that their null generator of the event horizon was aligned with Killing vector. The extension to electromagnetic case, subject to a certain inequality comprising norm of the Killing vector field and electric field, was provided in [39]. The ADM formalism applied to the problem enables to get rid of the aforementioned inequality [40,41]. It was claimed that the solution of Einstein-Yang-Mills is static if it had a vanishing Yang-Mills electric field on static slices of the considered spacetime. The enhancement of the staticity theorem was provided in Einstein-Maxwell-axiondilaton gravity being the low-energy limit of the heterotic string theory $[42,43]$, and in higher dimensional EinsteinMaxwell gravity [44]. Staticity theorem plays an important role from the point of view of the uniqueness theorem for black holes (classification of black holes). Namely in nonrotating case staticity is required, whereas uniqueness for stationary axisymmetric black hole solutions is established by demanding circularity. Both staticity and circularity imposes additional requirements on fields in the underlying spacetimes.

As far as the validity of the staticity in black hole mathematical theory is concerned, one should begin with a key result in the theory, which constitutes the theorem conceived by Hawking, the so-called strong rigidity theorem $[38,45]$. The power of it lies in the fact that it binds two distinct independently defined notions. The notion of black hole event horizon and a Killing vector field. This theorem is of the key importance in the classification of stationary black holes. In Ref. [46] a refinement of the proof of the strong rigidity theorem avoiding the previous assumption concerning maximal analytic extensions which were not unique, was elaborated. Further generalisation of the rigidity theoremfor a spacetime with a stationary event horizon or a compact Cauchy horizon was given in [47].

It turns out that the uniqueness theorems for black holes are based on stronger assumptions than the strong rigidity theorem. As was mentioned above in the non-rotating case one requires staticity whereas in the rotating case the uniqueness theorem is established for circular spacetimes. However, another problem concerning the staticity theorem is to establish the stationarity in the strict sense (strictly stationarity), i.e., one wants to exclude ergoregions in the non-rotating case [48-50].

The main aim of the uniqueness theorems of black holes in general relativity is to show that the static electrovac black hole spacetime are described by Reissner-Nordström 
spacetime whereas the circular one is diffeomorphic to KerrNewman spacetime. For the first time, in Ref. [51] the problem of classification of non-singular black hole solutions was studied. Then, in [52-54] it was shown how to weaken the original assumption presented in [51]. The most complete results were proposed in Refs. [55-59]. The classification of static vacuum and electro-vacuum black hole solutions was finished in $[60,61]$, while by taking into account the near-horizon geometry, the last restriction that all degenerate components of the event horizon should have charges of the same signs, was removed [62].

The uniqueness black hole theorem for stationary axisymmetric spacetime turned out to be far more complicated task. It was elaborated in Refs. [39,63], while the complete proof was found by Mazur [64] and Bunting [65].

On the other hand, the unification attempts such as $\mathrm{M} /$ string theory caused the resurgence of works devoted to the mathematical aspects of the low-energy string theory black holes as well as higher dimensional ones. Tthe uniqueness of the black hole solutions in dilaton gravity was proved in works $[66,67]$, while the uniqueness of the static dilaton $U(1)^{2}$ black holes being the solution of $N=4, d=4$ supergravity was provided in [68]. The extension of the proof to the theory to allow for the inclusion of $U(1)^{N}$ static dilaton black holes was established in [69].

The layout of the paper is as follows. In Sect. 2, having in mind the attitude presented in $[40,41]$, we provide some basic idea concerning the Arnowitt-Deser-Misner formulation of Einstein-Maxwell dark matter gravity. We find the Hamiltonian for the theory in question, by the Legendre transformation and give the evolution equations. In Sect. 3 one treats the problem of canonical energy and momentum, where the asymptotically flat hypersurface possesses and does not have the inner boundary. Section 4 is devoted to the mass formula and staticity theorem for charged dark matter gravity. We assume that the black hole in question will be stationary and has a bifurcate Killing horizon. The main aspect of these studies will be two-folded. Firstly we shall look for the mathematical aspect of the staticity theorem for the black holes influenced by the auxiliary $U(1)$-gauge field, responsible for the hidden sector. Secondly in the light of growing new astrophysical observations of black holes, we take into account the problem how dark matter will modify the black hole characteristics like mass and angular momentum of these objects.

\section{Arnowitt-Deser-Misner formalism for dark sector gravity}

In this section we shall provide the basic idea for the $(3+1)$ formalism for the Einstein-Maxwell dark matter theory. The formalism in question considers the four-dimensional geom- etry to consist of a foliation of three-geometries. It enables to divide the metric into spatial and temporal parts, i.e., the line element yields

$d s^{2}=-(N d t)^{2}+h_{a b}\left(d x^{a}+N^{a} d t\right)\left(d x^{b}+N^{b} d t\right)$,

where $N$ is the lapse function while $N^{a}$ stands for the shift vector for the constant time hypersurface in the underlying manifold. The geometry of the manifold can be described in terms of the intrinsic metric and the extrinsic curvature of a three-dimensional hypersurface, $N$ and $N^{a}$ relate the intrinsic coordinate on one hypersurface to the intrinsic coordinates on a nearby hypersurface. Spacetime is sliced into spacelike hypersurfaces with each hypersurface labeled by a global time parameter [70,71]. The general covariance allows the great arbitrariness in the choice of aforementioned functions $N^{\mu}=\left(N, N^{a}\right)$.

In the canonical formulation of the Einstein-Maxwell dark matter theory the point in the phase space corresponds to the specification of the fields $\left(h_{a b}, \pi^{a b}, \tilde{A}_{i}, \tilde{B}_{i}, E_{i}, B_{i}\right)$ on a three-dimensional $\Sigma$ manifold, where $h_{a b}$ denotes Riemannian metric on $\Sigma, \tilde{A}_{i}$ and $\tilde{B}_{i}$ are $U(1)$-gauge Maxwell and dark sector fields on the three-dimensional manifold, while $E_{i}$ and $B_{i}$ are respectively, Maxwell and dark matter electric fields in the evolved spacetime. They constitute tensor densities quantities and imply the following relations:

$E_{k}=\sqrt{h} F_{\mu k} n^{\mu}$

for the Maxwell electric field, while the dark matter sector electric field $B_{i}$ is provided by

$B_{k}=\sqrt{h} B_{\mu k} n^{\mu}$

where $n^{\mu}$ constitutes the unit normal timelike vector to the hypersurface $\Sigma$ in the underlying spacetime.

The Lagrangian of the Einstein-Maxwell dark matter theory is subject to the relation

$$
\begin{aligned}
& \mathcal{L}_{E M-\text { dark matter }} \\
& =N \sqrt{h}\left({ }^{(3)} R+K_{a b} K^{a b}-K^{2}-F_{a b} F^{a b}+\frac{2}{h} E_{i} E^{i}\right. \\
& \left.-B_{a b} B^{a b}+\frac{2}{h} B_{i} B^{i}-\alpha F_{a b} B^{a b}+\frac{2 \alpha}{h} E_{i} B^{i}\right),
\end{aligned}
$$

where $K_{a b}$ denotes the extrinsic curvature.

The corresponding field momenta are achieved by variation of the underlying Lagrangian with respect to $\nabla_{0} h_{a b}$, $\nabla_{0} \pi_{a b}, \nabla_{0} \tilde{A}_{i}, \nabla_{0} \tilde{B}_{i}$, where $\nabla_{0}$ denotes the derivative with respect to time-coordinate.

Performing the variations with respect to $\nabla_{0} h_{a b}$, one has that the momentum $\pi^{a b}$ canonically conjugate to a Rieman- 
nian metric can be expressed by means of the extrinsic curvature $K_{a b}$ of $\Sigma$ hypersurface

$\pi^{a b}=\sqrt{h}\left(K^{a b}-h^{a b} K\right)$.

On the other hand, the momenta canonically conjugate to the $U(1)$ gauge fields $\tilde{A}_{i}$ and $\tilde{B}_{i}$ are equal respectively to

$$
\begin{aligned}
& \pi_{(F)}^{k}=\frac{\delta \mathcal{L}_{E M-\text { dark matter }}}{\delta\left(\nabla_{0} \tilde{A}_{k}\right)}=4 E^{k}+2 \alpha B^{k}, \\
& \pi_{(B)}^{k}=\frac{\delta \mathcal{L}_{E M-\text { dark matter }}}{\delta\left(\nabla_{0} \tilde{B}_{i}\right)}=4 B^{k}+2 \alpha E^{k} .
\end{aligned}
$$

The Hamiltonian for the Einstein-Maxwell dark matter theory will be defined by the Legendre transform. It is given by

$$
\begin{aligned}
\mathcal{H} & =\pi^{i j} \nabla_{0} h_{i j}+\pi_{(F)}^{i} \nabla_{0} \tilde{A}_{i}+\pi_{(B)}^{i} \nabla_{0} \tilde{B}_{i}-\mathcal{L}_{E M-\text { dark matter }} \\
& =N^{\mu} C_{\mu}+\tilde{A}_{0} \tilde{\tilde{A}}+\tilde{B}_{0} \tilde{\tilde{B}}+\mathcal{H}_{\text {div }},
\end{aligned}
$$

where $\mathcal{H}_{\text {div }}$ is the total divergence and has the form as follows:

$\mathcal{H}_{\text {div }}=D_{k}\left(\tilde{A}_{0} \pi_{(F)}^{k}+\tilde{B}_{0} \pi_{(B)}^{k}\right)+2 D_{a}\left(\frac{N_{b} \pi^{a b}}{\sqrt{h}}\right)$,

where $D_{m}$ denotes the covariant derivative with respect to the metric $h_{a b}$, on the hypersurface $\Sigma$.

Because of the fact that the time component of $U(1)$ gauge fields $\tilde{A}_{0}, \tilde{B}_{0}$ do not posses associated kinetic terms, they can be regarded as Lagrange multipliers. They will be subject to the generalised Gauss laws provided by

$$
\tilde{\tilde{A}}=-D_{k} \pi_{(F)}^{k}=0, \quad \tilde{\tilde{B}}=-D_{k} \pi_{(B)}^{k}=0 .
$$

For the components $C^{\mu}$ one obtains the following expressions:

$$
\begin{aligned}
C_{0}= & \sqrt{h}\left(F_{a b} F^{a b}+B_{a b} B^{a b}+\alpha F_{a b} B^{a b}\right) \\
& +\frac{2}{\sqrt{h}}\left(E_{k} E^{k}+B_{k} B^{k}+\alpha E_{k} B^{k}\right) \\
& +\sqrt{h}\left(-{ }^{(3)} R+\frac{1}{h}\left(\pi_{i j} \pi^{i j}-\frac{1}{2} \pi^{2}\right)\right), \\
C_{a}= & 2\left(2 B_{a k} B^{k}+\alpha B_{a k} E^{k}+2 F_{a k} E^{k}+\alpha F_{a k} B^{k}\right) \\
& -2 \sqrt{h} D_{a}\left(\frac{N_{b} \pi^{a b}}{\sqrt{h}}\right) .
\end{aligned}
$$

It turns out that the evolution equations can be formally derived using the volume integral contribution to the Hamiltonian in questions, denoted by $\mathcal{H}_{v}$. It has pure constraint form, given by

$\mathcal{H}_{v}=\int_{\Sigma} d \Sigma N^{\mu} C_{\mu}$
To proceed further, let us implement the Hamiltonian principle and calculate the variations of the corresponding fields in the phase space of the of compact support. Finding arbitrary infinitesimal variations $\left(\delta h_{a b}, \delta \pi_{a b}, \delta \tilde{A}_{i}, \delta \tilde{B}_{i}, \delta E_{i}, \delta B_{i}\right)$, after integration by parts, we obtain the change of the Hamiltonian $\mathcal{H}_{v}$ caused by the variations in question. Namely, it yields

$$
\begin{aligned}
\delta \mathcal{H}_{v}= & \int_{\Sigma} d \Sigma\left(P^{a b} \delta h_{a b}+Q^{a b} \delta \pi_{a b}+R^{i} \delta \tilde{A}_{i}+P^{i} \delta \tilde{B}_{i}\right. \\
& \left.+S^{i} \delta E_{i}+Q^{i} \delta B_{i}\right),
\end{aligned}
$$

where we have denoted

$$
\begin{aligned}
P^{a b}= & N \sqrt{h} a^{a b}+2 N^{m}\left(2 B_{m}{ }^{a} B^{b}+\alpha B_{m}{ }^{a} E^{b}+2 F_{m}{ }^{a} E^{b}+\alpha F_{m}{ }^{a} B^{b}\right) \\
& +\sqrt{h}\left(h^{a b} D^{m} D_{m} N-D^{a} D^{b} N\right)-\mathcal{L}_{N^{i}} \pi^{a b} \\
Q_{a b}= & \frac{N}{\sqrt{h}}\left(2 \pi_{a b}-\pi_{k}{ }^{k} h_{a b}\right)+\mathcal{L}_{N^{i}} h_{a b} \\
R^{i}= & -4 \sqrt{h} D_{a}\left[N\left(F^{a i}+\frac{\alpha}{2} B^{a i}\right)\right]-4 \mathcal{L}_{N^{i}}\left(E^{i}+\frac{\alpha}{2} B^{i}\right) \\
P^{i}= & -4 \sqrt{h} D_{a}\left[N\left(B^{a i}+\frac{\alpha}{2} F^{a i}\right)\right]-4 \mathcal{L}_{N^{i}}\left(B^{i}+\frac{\alpha}{2} E^{i}\right) \\
S_{k}= & \frac{4 N}{\sqrt{h}}\left(E_{k}+\frac{\alpha}{2} B_{k}\right)+4\left(\mathcal{L}_{N^{i}} \tilde{A}_{k}+\frac{\alpha}{2} \mathcal{L}_{N^{i}} \tilde{B}_{k}\right)+2 D_{k}\left(N \tilde{A}_{0}\right) \\
& +\alpha D_{k}\left(N \tilde{B}_{0}\right) \\
Q_{k}= & \frac{4 N}{\sqrt{h}}\left(B_{k}+\frac{\alpha}{2} E_{k}\right)+4\left(\mathcal{L}_{N^{i}} \tilde{B}_{k}+\frac{\alpha}{2} \mathcal{L}_{N^{i}} \tilde{A}_{k}\right)+2 D_{k}\left(N \tilde{B}_{0}\right) \\
& +\alpha D_{k}\left(N \tilde{A}_{0}\right) .
\end{aligned}
$$

For the quantity $a^{a b}$ one has

$$
\begin{aligned}
a^{a b}= & \frac{1}{h}\left(2 \pi^{a}{ }_{j} \pi^{b j}-\pi_{m}{ }^{m} \pi^{a b}\right)-\frac{1}{2} h^{a b}\left(\pi_{i j} \pi^{i j}\right. \\
& \left.-\frac{1}{2} \pi_{m}{ }^{m} \pi_{k}{ }^{k}\right)+{ }^{(3)} R-\frac{1}{2} h^{a b(3)} R \\
& +2\left(F^{a j} F_{j}{ }^{b}+\frac{1}{4} h^{a b} F_{i j} F^{i j}\right)+2\left(B^{a j} B_{j}{ }^{b}+\frac{1}{4} h^{a b} B_{i j} B^{i j}\right) \\
& +2 \alpha\left(F^{a j} B_{j}{ }^{b}+\frac{1}{4} h^{a b} F_{i j} B^{i j}\right) \\
& +\frac{2}{h}\left(E^{a} E^{b}-\frac{1}{2} h^{a b} E_{k} E^{k}\right)+\frac{2}{h}\left(B^{a} B^{b}-\frac{1}{2} h^{a b} B_{k} B^{k}\right) \\
& +\frac{2 \alpha}{h}\left(E^{a} B^{b}-\frac{1}{2} h^{a b} E_{k} B^{k}\right) .
\end{aligned}
$$

$\mathcal{L}_{N^{i}} E^{k}$, as well as $\mathcal{L}_{N^{i}} B^{k}$ and $\mathcal{L}_{N^{i}} \pi^{a b}$ depicts the Lie derivatives of tensor densities and are given by

$$
\begin{aligned}
\mathcal{L}_{N^{i}} E^{k} & =\sqrt{h} N^{c} D_{c}\left(\frac{E^{k}}{\sqrt{h}}\right)-E^{c} D_{c} N^{k}+E^{k} D_{c} N^{c}, \\
\mathcal{L}_{N^{i}} B^{k} & =\sqrt{h} N^{c} D_{c}\left(\frac{B^{k}}{\sqrt{h}}\right)-B^{c} D_{c} N^{k}+B^{k} D_{c} N^{c}, \\
\mathcal{L}_{N^{i}} \pi^{a b} & =\sqrt{h} N^{c} D_{c}\left(\frac{\pi^{a b}}{\sqrt{h}}\right)-2 \pi^{c(a} D_{c} N^{b)}+\pi^{a b} D_{c} N^{c} .
\end{aligned}
$$


On the other hand, $\mathcal{L}_{N^{i}} \tilde{A}_{k}, \mathcal{L}_{N^{i}} \tilde{B}_{k}$ and $\mathcal{L}_{N^{i}} h_{a b}$ correspond to the ordinary Lie derivatives.

Having in mind the relation (15), we arrive at the following forms of the evolution equations for the Einstein-Maxwell dark matter system:

$$
\begin{aligned}
\dot{h}_{a b} & =\frac{\delta \mathcal{H}_{v}}{\delta \pi^{a b}}=Q_{a b}, \quad \dot{\pi}_{a b}=-\frac{\delta \mathcal{H}_{v}}{\delta h^{a b}}=-P_{a b}, \\
\dot{E}_{k} & =-\frac{\delta \mathcal{H}_{v}}{\delta \tilde{A}^{k}}=-R_{k}, \quad \dot{\tilde{A}}_{k}=\frac{\delta \mathcal{H}_{v}}{\delta E^{k}}=S_{k}, \\
\dot{B}_{k} & =-\frac{\delta \mathcal{H}_{v}}{\delta \tilde{B}^{k}}=-P_{k}, \quad \dot{\tilde{B}}_{k}=\frac{\delta \mathcal{H}_{v}}{\delta B^{k}}=Q_{k} .
\end{aligned}
$$

As was mentioned in $[40,41]$ the quantities $N^{\mu}, \tilde{A}_{0}, \tilde{B}_{0}$ are viewed as non-dynamical variables which are not represented in the phase space of Einstein-Maxwell dark matter theory. This fact enables us to choose them arbitrarily. The choice of $N^{\mu}$ is caused by the evolution of the considered system one looks for. In what follows we shall be interested in the case when $N^{\mu}$ will be connected with a time translation or rotation in the spacetime. On the other hand, $\tilde{A}_{0}$ and $\tilde{B}_{0}$ one restricts to the case of approaching angle-dependent quantities, when $r$-coordinates tends to infinity.

It worth mentioning that Eq. (15) corresponds to the volume contribution to the Hamiltonian of the theory in question. In the case when $\Sigma$ is a manifold without boundary, the nonvanishing surface terms should be taken into account, when the perturbations of $\mathcal{H}_{v}$ satisfying the asymptotic boundary conditions at infinity, are examined. They appear due to the integration by parts procedure. Just, if we want to put the Hamiltonian in question, into the form (14), one ought to get rid of the aforementioned surface terms by adding them with the opposite signs. Consequently, we arrive at

$$
\begin{aligned}
\mathcal{H}= & \mathcal{H}_{v}+\int_{S_{\infty}} d S_{k}\left[N\left(D^{a} h_{a}{ }^{k}-D^{k} h_{m}{ }^{m}\right)+2 \frac{N^{b} \pi^{k} b}{\sqrt{h}}\right. \\
& +4 N^{m}\left(\tilde{A}_{m}+\frac{\alpha}{2} \tilde{B}_{m}\right) E^{k}+2 N\left(\tilde{A}_{0}+\frac{\alpha}{2} \tilde{B}_{0}\right) E^{k} \\
& \left.+4 N^{m}\left(\tilde{B}_{m}+\frac{\alpha}{2} \tilde{A}_{m}\right) B^{k}+2 N\left(\tilde{B}_{0}+\frac{\alpha}{2} \tilde{A}_{0}\right) B^{k}\right] .
\end{aligned}
$$

By virtue of the direct calculation it can be envisaged that for all asymptotically flat perturbations, as well as, for $N^{\mu}, \tilde{A}_{0}, \quad \tilde{B}_{0}$ fulfilling the auxiliary conditions $r \rightarrow \infty$, one reaches the conclusion that variation $\delta \mathcal{H}$ is equal to the right-hand side of the relation given by the equation (15).

In what follows we shall examine the asymptotically flat initial data. Thus, one has that in an asymptotic region of hypersurface $\Sigma$, being diffeomorphic to $\mathbf{R}^{3}-B$, where $B$ is compact, the following fall-off conditions for the fields in the phase space will be fulfilled:

$$
\begin{aligned}
h_{a b} & \approx \delta_{a b}+\mathcal{O}\left(\frac{1}{r}\right), \\
\pi_{a b} & \approx \mathcal{O}\left(\frac{1}{r^{2}}\right), \\
\tilde{A}_{i} & \approx \mathcal{O}\left(\frac{1}{r}\right), \quad \tilde{B}_{i} \approx \mathcal{O}\left(\frac{1}{r}\right), \\
E_{i} & \approx \mathcal{O}\left(\frac{1}{r^{2}}\right), \quad B_{i} \approx \mathcal{O}\left(\frac{1}{r^{2}}\right) .
\end{aligned}
$$

Moreover, the standard asymptotic behavior of the lapse function, $N \approx 1+\mathcal{O}(1 / r)$, and shift function $N^{a} \approx \mathcal{O}(1 / r)$, will be supposed.

\section{Canonical energy, canonical momentum and the first law of thermodynamics}

In this section the problem of canonical energy and momentum with respect to the first law of black hole thermodynamics in Einstein-Maxwell dark matter sector gravity will be paid attention to. We shall scrutinise the case when the hypersurface $\Sigma$ being asymptotically flat has no inner boundary, as well as, the context of $\Sigma$ admits interior boundary.

\section{$3.1 \Sigma$ does not possess inner boundary}

To commence with let us define the canonical energy on the constrained submanifold of the considered phase space, as the Hamiltonian function bounded with the case when $N^{\mu}$ is an asymptotical translation at infinity. In the aforementioned case we have that, $N \rightarrow 1, N^{a} \rightarrow 0$. Consequently, using the exact form of the Hamiltonian (28) with the asymptotical conditions for $N^{\mu}$, it yields

$$
\mathcal{E}=\frac{1}{16 \pi} \mathcal{H}\left(N \rightarrow 1, N^{a} \rightarrow 0\right)=m+\mathcal{E}^{(F-\alpha)}+\mathcal{E}^{(B-\alpha)},
$$

where $m$ is the ADM mass, defined as follows:

$m=\frac{1}{16 \pi} \int_{S^{\infty}} d S_{k}\left(D^{a} h_{a}{ }^{k}-D^{k} h_{m}{ }^{m}\right)$,

while the quantities $\mathcal{E}^{(F-\alpha)}$ and $\mathcal{E}^{(B-\alpha)}$ imply

$$
\begin{aligned}
& \mathcal{E}^{(F-\alpha)}=\frac{1}{8 \pi} \int_{S^{\infty}} d S_{k}\left(\tilde{A}_{0}+\frac{\alpha}{2} \tilde{B}_{0}\right) E^{k}, \\
& \mathcal{E}^{(B-\alpha)}=\frac{1}{8 \pi} \int_{S^{\infty}} d S_{k}\left(\tilde{B}_{0}+\frac{\alpha}{2} \tilde{A}_{0}\right) B^{k} .
\end{aligned}
$$

One can also notice that $\mathcal{H}_{v}$, as a pure constraint on the considered phase space, disappears. 
The forms of $\mathcal{E}^{(F-\alpha)}$ and $\mathcal{E}^{(B-\alpha)}$ suggest that they are gauge dependent, because of the fact that both $\tilde{A}_{0}$ and $\tilde{B}_{0}$ may be chosen in an arbitrary way. However, as was stated in [40], if one takes into account a stationary solutions of Einstein-Maxwell dark matter equations of motion, these components of gauge fields are determined by the auxiliary conditions $\dot{\tilde{A}}_{k}=0, \dot{\tilde{B}}_{k}=0, \dot{E}_{k}=0, \dot{B}_{k}=0$, for all the cases when $N^{\mu}$ is chosen to be the stationary Killing vector field. For the choice of $\tilde{A}_{0}$, having in mind the Eq. (26) that $\dot{\tilde{A}}_{k}=0$, which in turn implies that $S_{k}=0$. Contracting this equation with $\tilde{A}_{0}+\alpha / 2 \tilde{B}_{0}$, we get

$$
2\left(\tilde{A}_{0}+\frac{\alpha}{2} \tilde{B}_{0}\right) D_{k}\left(N\left(\tilde{A}_{0}+\frac{\alpha}{2} \tilde{B}_{0}\right)\right)+\mathcal{O}\left(\frac{1}{r^{2}}\right)=0 .
$$

On the other hand, having in mind that $N \rightarrow 1$, one can write

$D_{k}\left[\left(\tilde{A}_{0}+\frac{\alpha}{2} \tilde{B}_{0}\right)^{2}\right]+\mathcal{O}\left(\frac{1}{r^{2}}\right)=0$.

It leads to the relation $\partial_{k}\left[\left(\tilde{A}_{0}+\frac{\alpha}{2} \tilde{B}_{0}\right)^{2}\right]=0$, which shows that the magnitude of $\tilde{A}_{0}+\frac{\alpha}{2} \tilde{B}_{0}$ is asymptotically constant. By virtue of this we define

$V_{(F-B)}=\lim _{r \rightarrow \infty} \sqrt{\tilde{A}_{0} \tilde{A}_{0}+\alpha \tilde{A}_{0} \tilde{B}_{0}+\frac{\alpha^{2}}{4} \tilde{B}_{0} \tilde{B}_{0} .}$

The same procedure applied to the dark matter sector $U(1)$ gauge field. Namely for the choice of $\tilde{B}_{0}$, Eq. (27) implies that $\dot{\tilde{B}}_{k}=0$, which leads to the conclusion that $Q_{k}$ is equal to zero. Contracting this relation with $\tilde{B}_{0}+\alpha / 2 \tilde{A}_{0}$, results in definition of the form as

$V_{(B-F)}=\lim _{r \rightarrow \infty} \sqrt{\tilde{B}_{0} \tilde{B}_{0}+\alpha \tilde{A}_{0} \tilde{B}_{0}+\frac{\alpha^{2}}{4} \tilde{A}_{0} \tilde{A}_{0}}$

To proceed further, let us contract $\dot{\tilde{A}}_{k}=0$ with $\tilde{A}_{0}+\frac{\alpha}{2} \tilde{B}_{0}$ and take the divergence of the outcome. One gets that

$D_{k} D^{k} \chi_{F}^{2}=\mathcal{O}\left(\frac{1}{r^{4}}\right)$

where we set

$\chi_{F}^{2}=V_{(F-B)}^{2}+\frac{C_{1}}{r}+\mathcal{O}\left(\frac{1}{r}\right)$.
$C_{1}$ stands for a constant. Next, let us apply $D_{r}$ to the Eq. (42) for $\chi_{F}^{2}$. It implies

$2 N\left(\tilde{A}_{0}+\frac{\alpha}{2} \tilde{B}_{0}\right) D_{r}\left(N\left(\tilde{A}_{0}+\frac{\alpha}{2} \tilde{B}_{0}\right)\right)=-\frac{C_{1}}{r^{2}}+\mathcal{O}\left(\frac{1}{r^{2}}\right)$.

Having in mind the exact form of the derivative $D_{r}$ from the relation $\dot{\tilde{A}}_{k}=0$, we finally arrive at the following:

$$
\left(\tilde{A}_{0}+\frac{\alpha}{2} \tilde{B}_{0}\right)\left(E_{r}+\frac{\alpha}{2} B_{r}\right)=\frac{C_{1}}{r^{2}}+\mathcal{O}\left(\frac{1}{r^{2}}\right) .
$$

Taking into account the Eq. (39), we can define in the stationary case $\mathcal{E}^{(F-\alpha)}=V_{(F-B)} Q_{F}$, where the charge [72] is of the form

$$
Q_{F}= \pm \frac{1}{4 \pi} \int_{S_{\infty}} d S\left|\left(E_{k}+\frac{\alpha}{2} B_{k}\right) r^{k}\right|,
$$

where $r^{k}$ stands for the unit radial vector and the choice of \pm signs depends on whether

$\lim _{r \rightarrow \infty} r^{2}\left(\tilde{A}_{0}+\frac{\alpha}{2} \tilde{B}_{0}\right)\left(E_{m}+\frac{\alpha}{2} B_{m}\right) r^{m}=C_{1}$

has the positive or negative value.

Consequently, the equation $\dot{\tilde{B}}_{k}=0$ and the procedure described above, reveal that we arrive to the following relations:

$\chi_{B}^{2}=V_{(B-F)}^{2}+\frac{C_{2}}{r}+\mathcal{O}\left(\frac{1}{r}\right)$,

where $C_{2}$ denotes a constant and the relation

$$
\left(\tilde{B}_{0}+\frac{\alpha}{2} \tilde{A}_{0}\right)\left(B_{r}+\frac{\alpha}{2} E_{r}\right)=\frac{C_{2}}{r^{2}}+\mathcal{O}\left(\frac{1}{r^{2}}\right) .
$$

All these enable us to define the charge connected with the dark matter sector

$Q_{B}= \pm \frac{1}{4 \pi} \int_{S_{\infty}} d S\left|\left(B_{k}+\frac{\alpha}{2} E_{k}\right) r^{k}\right|$,

with the choice of \pm signs depending on whether

$\lim _{r \rightarrow \infty} r^{2}\left(\tilde{B}_{0}+\frac{\alpha}{2} \tilde{A}_{0}\right)\left(B_{k}+\frac{\alpha}{2} E_{k}\right) r^{k}=C_{2}$

is of the positive or negative value.

Just considering initial data corresponding to a stationary solution to Einstein-Maxwell dark sector gravity and supposing that $N^{\mu}$ is stationary Killing vector field and choosing 
$\tilde{A}_{0}, \tilde{B}_{0}$ in such way that $\tilde{A}_{k}$ and $\tilde{B}_{k}$, as well as, $E_{k}$ and $B_{k}$ are time independent, we arrive at the following theorem:

Let us suppose that $\left(h_{a b}, \pi^{a b}, \tilde{A}_{i}, \tilde{B}_{i}, E_{i}, B_{i}\right)$ constitute smooth data for a stationary, asymptotically flat solution for Einstein-Maxwell dark matter system. Moreover, we assume that the hypersurface $\Sigma$ of the initial data set has no interior boundary and $\left(\delta h_{a b}, \delta \pi_{a b}, \delta \tilde{A}_{i}, \delta \tilde{B}_{i}, \delta E_{i}, \delta B_{i}\right)$ authorises smooth, asymptotically flat solution of linearised constraint relations for the underlying theory. Then one arrives at the following relation:

$\delta \mathcal{E}=\delta m+\delta \mathcal{E}^{(F-\alpha)}+\delta \mathcal{E}^{(B-\alpha)}=0$,

where $\delta \mathcal{E}^{(F-\alpha)}=V_{(F-B)} \delta Q_{F}$ and $\delta \mathcal{E}^{(B-\alpha)}=V_{(B-F)} \delta Q_{B}$.

One can conclude that every stationary solution is an extremum of the ADM mass at fixed both electric Maxwell and electric dark matter sector charges.

\section{2 $\Sigma$ possesses the inner boundary}

At first, we pay attention to the canonical angular momentum $\mathcal{J}$, defined as a Hamiltonian function being subject to the condition that $N^{\mu}$ is an asymptotical rotation. It this case $N \rightarrow 0$, while $N^{a} \rightarrow \phi^{a}$, where $\phi^{a}$ is the appropriate Killing vector field bounded with the rotation. On this account, it implies

$$
\begin{aligned}
\mathcal{J}= & -\frac{1}{16 \pi} \int_{S^{\infty}} d S_{k}\left[4 \phi^{m}\left(\tilde{A}_{m}+\frac{\alpha}{2} \tilde{B}_{m}\right) E^{k}\right. \\
& \left.+4 \phi^{m}\left(\tilde{B}_{m}+\frac{\alpha}{2} \tilde{A}_{m}\right) B^{k}+2 \phi^{m} \pi^{k}{ }_{m}\right] .
\end{aligned}
$$

If we convert the above integral into the one calculated over $\Sigma$, one gets

$$
\begin{aligned}
\mathcal{J}= & -\frac{1}{16 \pi} \int_{\Sigma} d \Sigma\left[\mathcal{L}_{\phi^{i}} h_{a b} \pi^{a b}+4 B^{k} \mathcal{L}_{\phi^{i}}\left(\tilde{B}_{k}+\frac{\alpha}{2} \tilde{A}_{k}\right)\right. \\
& \left.+4 E^{k} \mathcal{L}_{\phi^{i}}\left(\tilde{A}_{k}+\frac{\alpha}{2} \tilde{B}_{k}\right)\right]+\mathcal{J}_{\Sigma},
\end{aligned}
$$

where we set $\mathcal{J}_{\Sigma}$ in the form as follows:

$$
\begin{aligned}
\mathcal{J}_{\Sigma}= & -\frac{1}{16 \pi} \int d S_{k}\left[2 \phi^{m} \pi^{k}{ }_{m}+4 \phi^{m}\left(\tilde{A}_{m}+\frac{\alpha}{2} \tilde{B}_{m}\right) E^{k}\right. \\
& \left.+4 \phi^{m}\left(\tilde{B}_{m}+\frac{\alpha}{2} \tilde{A}_{m}\right) B^{k}\right] .
\end{aligned}
$$

In the relation (53) the integral over $\Sigma$ vanishes due to the fact that Killing vector field $\phi^{\mu}$ is equal to its tangential projection $[40,41]$. Thus if the hypersurface in question has no inner boundary the canonical momentum for the axisymmetric case is equal to zero.

Let us suppose that the hypersurface $\Sigma$ has an asymptotic region and a smooth interior boundary $S$. Moreover we set that $N \rightarrow 1, N^{a} \rightarrow \Omega \phi^{a}$, where $\phi^{a}$ is an axial Killing vector field, while $\Omega$ is a constant vale quantity. We obtain the following relation:

$$
\begin{aligned}
& 16 \pi(\delta \mathcal{E}-\Omega \delta \mathcal{J}) \\
& =\int_{\Sigma} d \Sigma\left(P^{a b} \delta h_{a b}+Q^{a b} \delta \pi_{a b}+R^{i} \delta \tilde{A}_{i}+P^{i} \delta \tilde{B}_{i}\right. \\
& \left.\quad+S^{i} \delta E_{i}+Q^{i} \delta B_{i}\right)+\delta(\text { surface terms }) .
\end{aligned}
$$

In [40], it was revealed that one can take an asymptotically flat hypersurface $\Sigma$, intersecting the sphere $S$ of a stationary black hole, being a bifurcation Killing horizon and taking into account $N^{\mu}$ equal to $\zeta^{\mu}=t^{\mu}+\Omega \phi^{\mu}$. Putting $\dot{\tilde{A}}_{k}=\dot{\tilde{B}}_{k}=0, \dot{E}_{k}=\dot{B}_{k}=0$, one finds that the integral of (55) disappears and only one surface term survives. This term is equal to $2 \pi \kappa \delta A$, where $\kappa$ stands for the surface gravity of the surface $S, A$ is the surface of $S$. Now, by the fact that the aforementioned Killing vector field responsible for the rotation, is equal to its tangential projection, the defined above angular momentum is equivalent to the angular momentum of black hole.

We conclude that in the case of $S$ being smooth inner boundary of the hypersurface $\Sigma$, one reaches the statement as follows:

\section{Theorem}

If $\left(h_{a b}, \pi^{a b}, \tilde{A}_{i}, \tilde{B}_{i}, E_{i}, B_{i}\right)$ comprises the smooth data for a stationary, asymptotically flat solution for a stationary black hole with bifurcation sphere lying on $\Sigma$, as well as, $\left(\delta h_{a b}, \delta \pi_{a b}, \delta \tilde{A}_{i}, \delta \tilde{B}_{i}, \delta E_{i}, \delta B_{i}\right)$ comprehends smooth, asymptotically flat solution of linearized constraint relations for the underlying theory, then

$\delta \mathcal{E}=\delta m+V_{(F-B)} \delta Q_{F}+V_{(B-F)} \delta Q_{B}-\Omega \delta \mathcal{J}=\frac{1}{8 \pi} \kappa \delta A$.

The relation (56) encompasses the first law of charged dark sector black hole thermodynamics.

\section{Mass formula and staticity theorem for dark matter black holes}

In this section we shall elaborate the problem of mass for black holes and staticity in Einstein-Maxwell dark matter gravity, in the spacetime representing a stationary black hole with a bifurcate Killing horizon with a bifurcation sphere.

In our studies one will pay attention to the stationary black hole with a bifurcate Killing horizon possesses a timelike Killing vector field, approaching a time translation in the asymptotic region and Killing vector field $\zeta^{\mu}$ which is orthogonal to the bifurcation sphere and vanishes on it. If $t^{\mu}$ fails to coincide with $\zeta^{\mu}$, then the spacetime has an addi- 
tional, axial Killing vector field $\phi^{\mu}$, satisfying

$\zeta^{\mu}=t^{\mu}+\Omega \phi^{\mu}$,

where $\Omega$ is the angular velocity of the black hole horizon, while the angular momentum of the black hole is defined respectively as

$I_{B H}=\frac{1}{16 \pi} \int d S^{\mu v} \epsilon_{\mu \nu \rho \sigma} \nabla^{\rho} \phi^{\sigma}$.

The constraints of the aforementioned spacetime theory will be given on hypersurface $\Sigma$ being the maximal one, i.e. for which one has that $\pi_{a}{ }^{a}=0$. The possibility of implementing such condition results from the theorem proved in [73], stating that any stationary black hole solution with bifurcate Killing horizon admits an asymptotically flat maximal hypersurface which is asymptotically orthogonal to $t^{\mu}$. Its boundary constitutes a bifurcate surface of the horizon.

To commence with, let us find for the hypersurface in question, the equation for the lapse function $N=-k^{\mu} n_{\mu}$, for any Killing vector $k_{\mu}$ and $n_{\mu}$ unit normal to the maximal hypersurface. Having in mind the equation for $C_{0}$, given by (12), it may be verified that

$D_{m} D^{m} N=\rho_{D} N$,

where for the explicit form of $\rho_{D}$ one obtains

$$
\begin{aligned}
\rho_{D}= & \frac{\pi_{a b} \pi^{a b}}{h}+\frac{1}{2}\left(F_{i j} F^{i j}+B_{i j} B^{i j}+\alpha F_{i j} B^{i j}\right) \\
& +\frac{1}{h}\left(E_{k} E^{k}+B_{k} B^{k}\right)+\frac{\alpha}{h} E_{k} B^{k} .
\end{aligned}
$$

In the next step, we choose $N=\lambda=-t^{\mu} n_{\mu}$. It satisfies the boundary conditions $\left.\lambda\right|_{S}=0$ and $\left.\lambda\right|_{\infty}=1$ By virtue of the maximal principle and integrating (59), one reach to the relation [41]

$4 \pi m-\kappa A=\int_{\Sigma} d \Sigma \lambda \rho_{D}$.

The above equation is the restriction for the mass of stationary black hole with bifurcate horizon in charged dark sector gravity.

From the explicit form of $\rho_{D}$ given by Eq. (60), one can see that there are additional terms (comparing to EinsteinMaxwell gravity) connected with dark sector, in the part encompasses strength and electric fields of the gauge fields in question. Let us analyze the sign of $\rho_{D}$. Having in mind a general inequality inequality of the form $\left(F_{i j}-B_{i j}\right)\left(F^{i j}-\right.$ $\left.B^{i j}\right) \geq 0$, one can deduce that $F_{i j} F^{i j}+B_{i j} B^{i j} \geq \alpha F_{i j} B^{i j}$. On the other hand, for the third and fourth term on the righthand side of (60), considering the inequality $\left(E_{i}-B_{i}\right)\left(E^{i}-\right.$
$\left.B^{i}\right) \geq 0$, one can show that $E_{i} E^{i}+B_{i} B^{i} \geq \alpha E_{i} B^{i}$. All these lead to the conclusion that $\rho_{D}$ is non-negative.

On the other hand, the generalisation of the above derivation, using the Raychaudhuri equation [45], reveals that in any spacetime being foliated by maximal hypersurfaces the lapse function fulfils the relation (59), with $\rho_{D}$

$\rho_{D}=\frac{1}{h} \pi_{i j} \pi^{i j}+R_{\mu \nu} n^{\mu} n^{\nu}$.

Further it implies that for a stationary black hole with a bifurcate horizon, Eq. (59) is satisfied and $\rho_{D}$ is given by (62). Moreover $\rho_{D}$ will be non-negative provided that the matter fields satisfy the strong energy condition [41]. It turned out that when $\rho_{D}$ is non-negative the maximum principle may be implemented and solutions of (59) are uniquely determined by their boundary and asymptotic values.

Dark matter sector intensively influences on black hole mass, not only as the auxiliary field can contribute (as it is expected from the outset) but one can see that the kinetic mixing term plays also the significant role in the mass of the black objects. Perhaps this fact may justify the existence of supermassive black holes in the early Universe, when dark matter was in abundance and played the significant role in the formation of cosmic web on which the ordinary matter accumulated. As far as the observation of the objects in question is concerned, recently it has been announced [74] that 83 new supermassive black holes have been discovered, at the distances when the Universe was only $5 \%$ of its current age. The formation of such massive objects soon after the Big Bang is still a tentative question and a real challenge for contemporary astrophysics and cosmology.

The similar results concerning the significant influence of the hidden sector on black hole mass have been revealed in [75], where the physical version of the first law of black hole and first law of mechanics for the compact binary system is considered. On the other hand, the exact metric of static spherically symmetric solution in Einstein-Maxwell charged dark matter gravity was obtained in [76].

Inspection of the Eq. (61) reveals that because of the fact that $\lambda, \rho_{D}$ are non-negative quantities, as in the case of Einstein Yang-Mills [41], Einstein-Maxwell-axion-dilaton gravity being the low-energy limit of the heterotic string theory [43], higher-dimensional Einstein-Maxwell gravity [44], we obtain the conclusion that mass of the black hole is greater or equal to the area of the bifurcate sphere multiplied by the surface gravity. The non-rotating case was treated in [77], where the inequality of this kind was revealed.

The other way of examining the mass formula is to exploit the relation derived in [78]

$$
m=2 \int_{\Sigma} d \Sigma\left(T_{\mu \nu}-\frac{1}{2} g_{\mu \nu} T\right) t^{\mu} n^{\nu}+\frac{\kappa A}{4 \pi}+2 \Omega I_{B H}
$$


Implementing the exact form of the energy momentum tensor one obtains

$$
\begin{aligned}
m- & \frac{\kappa A}{4 \pi}-2 \Omega I_{B H} \\
= & \frac{1}{4 \pi} \int_{\Sigma} d \Sigma\left[\frac{2 t^{m}}{\sqrt{h}}\left(F_{m d} E^{d}+B_{m d} B^{d}+\alpha F_{m d} B^{d}\right)\right. \\
& +\frac{\lambda}{2}\left(F_{a b} F^{a b}+B_{a b} B^{a b}+\alpha F_{a b} B^{a b}\right) \\
& \left.+\frac{\lambda}{h}\left(E_{i} E^{i}+B_{i} B^{i}+\alpha E_{i} B^{i}\right)\right]
\end{aligned}
$$

where $\lambda=-t^{\mu} n_{\mu}$.

One should remark that the integral over $\Sigma$ in Eq. (53) vanishes, due to the fact that axial Killing field $\phi^{\mu}$ is equal to its projection [41]. This fact implies that in our case we obtain that

$\mathcal{J}=\mathcal{J}_{\Sigma}$

Having in mind (52) and (53) and the relation [41] $\nabla^{\mu} \phi^{\nu}=D^{\mu} \phi^{\nu}-2 \phi_{\rho} n^{[\mu} K^{\nu] \rho}$, where $K_{\mu \nu}$ is the extrinsic curvature of the hypersurface $\Sigma$, one can draw a conclusion that $1 / 8 \pi \int_{S} d S_{a} \pi^{a b} \phi_{b}$ is equal to the black hole angular momentum $I_{B H}$. Computing the second term in (52), we reveal that

$$
\begin{aligned}
& 4 \pi \Omega\left(I_{B H}-\mathcal{J}_{\Sigma}\right) \\
& =-\int_{S} \frac{d S_{k}}{\sqrt{h}}\left[t^{m}\left(\tilde{A}_{m}+\frac{\alpha}{2} \tilde{B}_{m}\right) E^{k}+t^{m}\left(\tilde{B}_{m}+\frac{\alpha}{2} \tilde{A}_{m}\right) B^{k}\right] .
\end{aligned}
$$

Consequently, for $N=\lambda$ we finally find that the following is fulfilled:

$$
\begin{aligned}
4 \pi & \left(V_{(F-B)} Q_{F}+V_{(B-F)} Q_{B}\right)+4 \pi \Omega\left(I_{B H}-\mathcal{J}_{\Sigma}\right) \\
= & \int_{\Sigma} \frac{d \Sigma}{\sqrt{h}}\left[-\frac{\lambda}{4 \sqrt{h}}\left(4 E_{k}+2 \alpha B_{k}\right) E^{k}+t^{m}\left(F_{k m}+\frac{\alpha}{2} B_{k m}\right) E^{k}\right] \\
& +\int_{\Sigma} \frac{d \Sigma}{\sqrt{h}}\left[-\frac{\lambda}{4 \sqrt{h}}\left(4 B_{k}+2 \alpha E_{k}\right) B^{k}+t^{m}\left(B_{k m}+\frac{\alpha}{2} F_{k m}\right) B^{k}\right] .
\end{aligned}
$$

On the other hand, the relations (61) and (67) and using the exact form of $\rho_{D}$, one receives the following:

$$
\begin{aligned}
& 8 \pi\left[\Omega \mathcal{J}_{\Sigma}-\left(V_{(F-B)} Q_{F}+V_{(B-F)} Q_{B}\right)\right] \\
& =\int_{\Sigma} d \Sigma \frac{\lambda}{h}\left[\pi_{a b} \pi^{a b}+2\left(E_{i}+\frac{\alpha}{2} B_{i}\right) E^{i}\right. \\
& \left.\quad+2\left(B_{i}+\frac{\alpha}{2} E_{i}\right) B^{i}\right] .
\end{aligned}
$$

As in [41], we remark that the exterior region of dark matter sector black hole can be foliated by maximal hypersurfaces with a boundary $S$. They are asymptotically orthogonal to the timelike Killing vector field $t^{\mu}$, when the strong energy condition is satisfied. Making use the equation (68) to such kind of hypersurfaces, we have that $\pi^{a b}=0$, and $E_{k}=$ $B_{k}=0$. Consequently, we get that $\lambda n^{\mu}$ is a Killing vector and $t^{\mu}=\lambda n^{\mu}$. It provides the following theorem:

The stationary black hole solution of Einstein-Maxwell dark matter system with bifurcate Killing horizon satisfying the condition

$\Omega \mathcal{J}_{\Sigma}-\left(V_{(F-B)} Q_{F}+V_{(B-F)} Q_{B}\right)=0$,

is static and possesses vanishing electric Maxwell $E_{k}$ and dark matter electric $B_{k}$, fields on static slices through the underlying spacetime.

Inspection of the Eqs. (61), (67) and the relation

$m+V_{(F-B)} Q_{F}+V_{(B-F)} Q_{B}-2 \Omega \mathcal{J}_{\Sigma}=\frac{\kappa A}{4 \pi}$,

which follows from (56) and the scaling properties [40] of $m \rightarrow \beta m, V_{(F-B)} \rightarrow V_{(F-B)}, V_{(B-F)} \rightarrow V_{(B-F)}, \Omega \rightarrow$ $1 / \beta \Omega, \mathcal{J}_{\Sigma} \rightarrow \beta^{2} \mathcal{J}_{\Sigma}, \kappa \rightarrow 1 / \beta \kappa, A \rightarrow \beta^{2} A, \tilde{A}_{\mu} \rightarrow$ $\beta \tilde{A}_{\mu}, \tilde{B}_{\mu} \rightarrow \beta \tilde{B}_{\mu}$, reveals that

$$
\begin{aligned}
8 \pi \Omega \mathcal{J}_{\Sigma}= & \int_{\Sigma} d \Sigma \lambda\left(F_{a b} F^{a b}+B_{a b} B^{a b}+\alpha F_{a b} B^{a b}\right) \\
& +\int_{\Sigma} d \Sigma \frac{\lambda}{h} \pi_{a b} \pi^{a b}
\end{aligned}
$$

and

$$
\begin{aligned}
& 8 \pi\left(V_{(F-B)} Q_{F}+V_{(B-F)} Q_{B}\right) \\
& =\int_{\Sigma} d \Sigma \lambda\left(F_{a b} F^{a b}+B_{a b} B^{a b}+\alpha F_{a b} B^{a b}\right) \\
& \quad-2 \int_{\Sigma} d \Sigma \frac{\lambda}{h}\left[\left(E_{i}+\frac{\alpha}{2} B_{i}\right) E^{i}+2\left(B_{i}+\frac{\alpha}{2} E_{i}\right) B^{i}\right] .
\end{aligned}
$$

Now we can formulate the staticity condition for EinsteinMaxwell dark matter gravity black holes.

\section{Theorem}

A solution of Einstein-Maxwell dark matter gravity (with vanishing magnetic charges in visible and hidden sectors) describing a stationary black hole with a bifurcation Killing horizon with a bifurcation sphere, for which $\Omega \mathcal{J}_{\Sigma}=0$ is static, being subject to the condition of vanishing Maxwell magnetic and dark matter magnetic fields on a static slices of the considered spacetime manifold.

\section{Discussion and conclusions}

In our paper we have studied the $(3+1)$ formulation of the charged dark matter gravity, being generalisation of Einstein-Maxwell theory with additional $U(1)$-gauge field 
coupled to the ordinary Maxwell one, by the so-called kinetic mixing term. In this so-called dark photon model, the auxiliary field represents the hidden sector and mimicked the properties of dark matter.

The Arnowitt-Deser-Misner formalism enables one to find the variations of mass, angular momentum and canonical energy for the spacetime possessing the inner boundary and without it. If the inner boundary is a spacetime of topology of a sphere $S^{2}$, we have found the generalised black hole first law of thermodynamics in which the terms connected with the hidden sector play the crucial role.

Inspection of the Eqs. (60), (61) and (64), leads us to the conclusion that for the stationary black hole with a bifurcate Killing horizon, dark matter sector plays the significant role for value of its mass. The present results reveal that hidden sector not only contribute to the black hole mass as the auxiliary field can do (as it is expected from the outset) but also via kinetic mixing term proportional to $F_{a b} B^{a b}$.

Maybe, due to the large abundance of dark matter in our Universe, this fact may contribute to the explanation of the existence of supermassive black holes in the early Universe, when dark matter played the significant role in construction of the scaffolding (cosmic web) on which the ordinary matter condensed. Black objects might gain their masses via collapse of ordinary matter and hidden sector fields coupled in non-trivial way to Maxwell one.

Elaborating the maximal initial data for the aforementioned theory, we have found that stationary black hole being the solution of Einstein-Maxwell dark matter gravity, with bifurcate Killing horizon, satisfying the relation

$\Omega \mathcal{J}_{\Sigma}-\left(V_{(F-B)} Q_{F}+V_{(B-F)} Q_{B}\right)=0$,

is static and possesses vanishing both Maxwell electric and dark matter electric fields on a static slices of the spacetime. On the other hand, the black hole for which $\Omega \mathcal{J}_{\Sigma}=0$, is static being subject to disappearing both Maxwell and dark matter magnetic fields on static slices of the manifold in question.

These auxiliary conditions imposed on the adequate electric or magnetic components of the gauge fields, in the sense of definitions (3) and (4), constitute the so-called staticity conditions for charged dark matter gravity enabling to achieve the static black hole solution in the theory.

The next aim will be to implement these restrictions to prove the uniqueness of static charged black holes with the hidden sector field (find their classification), as well as, to consider the next step in construction of uniqueness theorem for stationary axisymmetric black hole solutions, circularity theorem. We hope to return to these subjects elsewhere.
Data Availability Statement This manuscript has no associated data or the data will not be deposited [Author's comment: The article concerns entirely theoretical researches].

Open Access This article is licensed under a Creative Commons Attribution 4.0 International License, which permits use, sharing, adaptation, distribution and reproduction in any medium or format, as long as you give appropriate credit to the original author(s) and the source, provide a link to the Creative Commons licence, and indicate if changes were made. The images or other third party material in this article are included in the article's Creative Commons licence, unless indicated otherwise in a credit line to the material. If material is not included in the article's Creative Commons licence and your intended use is not permitted by statutory regulation or exceeds the permitted use, you will need to obtain permission directly from the copyright holder. To view a copy of this licence, visit http://creativecomm ons.org/licenses/by/4.0/.

Funded by $\mathrm{SCOAP}^{3}$.

\section{References}

1. R. Massey et al., Dark matter maps reveal cosmic scaffolding. Nature 445, 286 (2007)

2. J. Dietrich et al., A filament of dark matter between two cluster of galaxies. Nature 487, 202 (2012)

3. M. Regis, J.Q. Xia, A. Cuoso, E. Branchini, N. Fornengo, M. Viel, Particle dark matter searches outside the local group. Phys. Rev. Lett. 114, 241301 (2015)

4. Y. Ali-Haimoud, J. Chluba, M. Kamionkowski, Constrants on dark matter interactions with standard model particles from cosmic microwave background spectral distortions. Phys. Rev. Lett. 115, 071304 (2015)

5. R. Bernabei, Searching for WIMPs by the annual modulation signature. Phys. Lett. B 424, 195 (1998)

6. R. Bernabei et al., Final model independent result of DAMA/LIBRA-phase1. Eur. Phys. J. C 73, 2648 (2013)

7. The COSINE-100 Collaboration, An experiment to search for darkmatter interactions using sodium iodide detectors, Nature 564, 83 (2018)

8. N. Masi, Dark matter: TeV-ish rather than miraculous, collisionless rather than dark. Eur. Phys. J. Plus 130, 69 (2015)

9. J.P. Lees et al., Search for a dark photon in $e^{+} e^{-}$collisions at BABAR. Phys. Rev. Lett. 113, 201801 (2014)

10. T.G. Rizzo, Kinetic mixing, dark photons and an extra dimension. Part I. JHEP 07, 118 (2018)

11. A. Geringer-Sameth, M.G. Walker, Indication of Gamma-ray emission from the newly discovered Dwarf Galaxy Reticulum II. Phys. Rev. Lett. 115, 081101 (2015)

12. K.K. Boddy, J. Kumar, Indirect detection of dark matter using MeVrange gamma-rays telescopes. Phys. Rev. D 92, 023533 (2015)

13. K. Tilburg, N. Leefer, L. Bougas, D. Budker, Search for ultralight scalar dark matter with atomic spectroscopy. Phys. Rev. Lett. 115, 011802 (2015)

14. J.H. Chang, R. Essig, S.D. McDermott, Revisiting Supernova 1987A constraints on dark photons. JHEP 01, 107 (2017)

15. H.-K. Guo, Y. Ma, J. Shu, X. Xue, Q. Yuan, Y. Zhao, Detecting dark photon matter with Gaia-like astrometry observations. JCAP 05, 015 (2019)

16. D. Harvey, R. Massey, T. Kitching, A. Taylor, E. Tittley, The nongravitational interactions of dark matter in colliding galaxy clusters. Science 347, 1462 (2015)

17. O. Abramoff et al., SENSEI: direct-detection constraints on sub$\mathrm{GeV}$ dark matter from a shallow underground run using a prototype skipper CCD. Phys. Rev. Lett. 122, 161801 (2019) 
18. G. Bertone, T.M.P. Tait, A new era in the search for dark matter. Nature 562, 51 (2018)

19. T. Vachaspati, A. Achucarro, Semilocal cosmic strings. Phys. Rev. D 44, 3067 (1991)

20. A. Achucarro, T. Vachaspati, Semilocal and electroweak strings. Phys. Rep. 327, 347 (2000)

21. S.A. Abel, B.W. Schofield, Brane-antibrane kinetic mixing, millicharged particles and SUSY breaking. Nucl. Phys. B 150, 685 (2004)

22. S.A. Abel, J. Jaeckel, V.V. Khoze, A. Ringwald, Illuminating the hidden sector of string theory by shining light through a magnetic field. Phys. Lett. B 666, 66 (2008)

23. S.A. Abel, M.D. Goodsell, J. Jaeckel, V.V. Khoze, A. Ringwald, Kinetic mixing of the photon with hidden U(1)s in string phenomenology. JHEP 07, 124 (2008)

24. D. Banerjee et al., Search for invisible decays of sub-GeV dark photons in missing-energy events at the CERN SPS. Phys. Rev. Lett. 118, 011802 (2017)

25. B.S. Acharya, S.A.R. Ellis, G.L. Kane, B.D. Nelson, M.J. Perry, Lightest visible-sector supersymmetric particle is likely unstable. Phys. Rev. Lett. 117, 181802 (2016)

26. B. Holdom, Two U(1)'s and $\epsilon$ charge shifts. Phys. Lett. B 166, 196 (1986)

27. D. Lüst, Intersecting brane worlds: a path to the standard model? Class. Quantum Grav. 21, S1399 (2004)

28. S. Abel, J. Santiago, Constraining the string scale: from Planck to weak and back again. J. Phys. G 30, R83 (2004)

29. S.A. Abel, M.D. Goodsell, J. Jaceckel, V.V. Khoze, A. Ringwald, Kinetic mixing term of with hidden U(1)s in string phenomenology. JHEP 07, 124 (2008)

30. P. Jean et al., Early SPI/INTEGRAL measurements of $511 \mathrm{keV}$ line emission from the 4th quadrant of the Galaxy. Astron. Astrophys. 407, L55 (2003)

31. J. Liu, N. Weiner, W. Xue, Signals of a light dark force in the galactic center. JHEP $\mathbf{0 8}, 050$ (2015)

32. J. Chang et al., An excess of cosmic ray electrons at energies of 300-800 GeV. Nature 456, 362 (2008)

33. O. Adriani et al. (PAMELA Collaboration), An anomalous positron abundance in cosmic rays with energies $1.5-100 \mathrm{Gev}$. Nature 458, 607 (2009)

34. G.W. Bennett et al., Final report of the E821 muon anomalous magnetic moment measurement at BNL. Phys. Rev. D 73, 072003 (2006)

35. H. Davoudiasl, H.S. Lee, W.J. Marciano, "Dark" Z implications for parity violation, rare meson decays, and Higgs physics. Phys. Rev. D 85, 115019 (2012)

36. H. Davoudiasl, H.S. Lee, I. Lewis, W.J. Marciano, Higgs decays as a window into the dark sector. Phys. Rev. D 88, 015022 (2013)

37. A. Lichnerowicz, Theories Relativistes de la Gravtation et de l'Electromagnetism (Mason, Paris, 1955)

38. S.W. Hawking, Black holes in general relativity. Commun. Math. Phys. 25, 152 (1972)

39. B. Carter, in Black Holes, ed. by C. DeWitt, B.S. DeWitt (Gordon and Breach, New York, 1973)

40. D. Sudarsky, R. Wald, Extrema of mass, stationarity, and staticity, and solutions to the Einstein-Yang-Mills equations. Phys. Rev. D 46, 1453 (1992)

41. D. Sudarsky, R. Wald, Mass formula for stationary Einstein-Yang Mills black holes and a simple proof of two staticity theorems. Phys. Rev. D 47, R5209 (1993)

42. M. Rogatko, The staticity problem for non-rotating black holes in Einstein-Maxwell axion-dilaton gravity. Class. Quantum Grav. 14, 2425 (1997)

43. M. Rogatko, Extrema of mass, first law of black hole mechanics, and a staticity theorem in Einstein-Maxwell-axion-dilaton gravity. Phys. Rev. D 58, 044011 (1998)
44. M. Rogatko, Staticity theorem for a higher dimensional generalized Einstein-Maxwell system. Phys. Rev. D 71, 024031 (2005)

45. S.W. Hawking, G.F.R. Ellis, The Large Scale Structure of SpaceTime (Cambridge University Press, Cambridge, 1973)

46. P.T. Chruściel, On rigidity on analytic black holes. Commun. Math. Phys. 189, 1 (1997)

47. H. Friedrich, I. Racz, R.M. Wald, On the rigidity theorem for spacetimes with a stationary event horizon or a compact Cauchy horizon. Commun. Math. Phys. 204, 691 (1999)

48. P. Haijcek, General theory of vacuum ergospheres. Phys. Rev. D 7, $2311(1973)$

49. P. Haijcek, Stationary electrovacuum spacetimes with bifurcate horizons. J. Math. Phys. 16, 518 (1975)

50. P. Haijcek, Static electrovacuum spacetimes with bifurcate horizons. J. Math. Phys. 16, 523 (1975)

51. W. Israel, Event horizons in static vacuum space-times. Phys. Rev. 164, 1776 (1967)

52. H. Müller zum Hagen, C.D. Robinson, H.J. Seifert, Black holes in static vacuum space-times. Gen. Relativ. Gravit. 4, 53 (1973)

53. H. Müller zum Hagen, C.D. Robinson, H.J. Seifert, Black holes in static electrovac space-times. Gen. Relativ. Gravit. 5, 61 (1974)

54. C.D. Robinson, A simple proof of the generalization of Israel's theorem. Gen. Relativ. Gravit. 8, 695 (1977)

55. G.L. Bunting, A.K.M. Masood-ul-Alam, Nonexistence of multiple black holes in asymptotically Euclidean static vacuum space-time. Gen. Relativ. Gravit. 19, 147 (1987)

56. P. Ruback, A new uniqueness theorem for charged black holes. Class. Quantum Grav. 5, L155 (1988)

57. A.K.M. Masood-ul-Alam, Uniqueness proof of static charged black holes revisited. Class. Quantum Grav. 9, L53 (1992)

58. M. Heusler, On the uniqueness of the Reissner-Nordström solution with electric and magnetic charge. Class. Quantum Grav. 11, L49 (1994)

59. M. Heusler, Staticity and uniqueness of multiple black hole solutions of sigma-models. Class. Quantum Grav. 10, 791 (1993)

60. P.T. Chruściel, The classification of static vacuum spacetimes containing an asymptotically flat spacelike hypersurface with compact interior. Class. Quantum Grav. 16, 661 (1999)

61. P.T. Chruściel, Towards a classification of static electrovacuum spacetimes containing an asymptotically flat spacelike hypersurface with compact interior. Class. Quantum Grav. 16, 689 (1999)

62. P.T. Chruściel, P. Tod, The classification of static electro-vacuum spacetime containing an asymptotically flat spacelike hypersurface with compact interior. Commun. Math. Phys. 271, 577 (2007)

63. C.D. Robinson, Uniqueness of the Kerr black hole. Phys. Rev. Lett. 34, 905 (1975)

64. P.O. Mazur, Proof of uniqueness of the Kerr-Newman black hole solution. J. Phys. A Math. Gen. 15, 3173 (1982)

65. G.L. Bunting, Ph.D. thesis, University of New England, Armidale, NSW (1983)

66. A.K.M. Masood-ul-Alam, Uniqueness of a static charged dilaton black hole. Class. Quantum Grav. 10, 2649 (1993)

67. M. Mars, W. Simon, On uniqueness of static Einstein-Maxwelldilaton black holes. Adv. Theor. Math. Phys. 6, 279 (2002)

68. M. Rogatko, Uniqueness theorem for static dilaton $\mathrm{U},(1)^{2}$ black holes. Phys. Rev. D 59, 104010 (1999)

69. M. Rogatko, Uniqueness theorem for static dilaton $U(1)^{N}$ black holes. Class. Quantum Grav. 19, 875 (2002)

70. R. Arnowitt, S. Deser, C.W. Misner, in Gravitation: An Inntroduction to Current Research, ed. by L. Witten (Willey, New York, 1962)

71. R. Wald, General Relativity (Chicago University Press, Chicago, 1984)

72. P.T. Chruściel, W. Kondracki, Some global charges in classical Yang-Mills theory. Physics 36, 1874 (1987) 
73. P.T. Chruściel, R. Wald, Maximal hypersurfaces in stationary asymptotically flat spacetimes. Commun. Math. Phys. 163, 561 (1994)

74. Y. Matsuoka et al., Discovery of the first low-luminosity quasar at $z>7$. Astrophys. J. Lett. 872, L2 (2019)

75. M. Rogatko, A. Wojnar, Dark matter influence on black holes thermodynamics. JCAP 05, 023 (2018)
76. B. Kiczek, M. Rogatko, Ultra-compact spherically symmetric dark matter charged star objects. JCAP 09, 049 (2019)

77. M. Visser, Dirty black holes: thermodynamics and horizon structure. Phys. Rev. D 46, 2445 (1992)

78. J.M. Bardeen, B. Carter, S.W. Hawking, The four laws of black hole mechanics. Commun. Math. Phys. 31, 161 (1973) 